\title{
Can myasthenia gravis be diagnosed with the 'ice pack test'? A cautionary note
}

\author{
Andrew J Larner, Dafydd J Thomas
}

\begin{abstract}
Summary
The ice pack test may be helpful in establishing that ptosis is due to ocular myasthenia gravis, since cold improves neuromuscular transmission. However, the role of the test in determining whether diplopia is of myasthenic origin has yet to be established.
\end{abstract}

Keywords: myasthenia gravis; diplopia; ice pack test

Myasthenia gravis often presents with ocular features, most usually diplopia and ptosis; $40 \%$ of patients have extra-ocular muscle involvement at onset and the disease remains confined to the eyes in $16 \%$ of patients. Establishing the diagnosis of isolated ocular myasthenia is sometimes difficult: symptomatic response to acetylcholinesterase medication may be equivocal, and acetylcholine receptor ( $\mathrm{AChR}$ ) auto-antibodies may be absent in up to $30 \%$ of those with symptoms restricted to the eyes for more than 2 years. Although electrophysiological evidence of neuromuscular junction transmission defects may be found in other muscles, this is also not always the case. ${ }^{1}$

The clinical observation that myasthenic symptoms may improve with cold and worsen with heat, ${ }^{2}$ and the electrophysiological finding that neuromuscular transmission may improve with local cooling, ${ }^{3}$ form the theoretical rationale for the use of the 'ice pack test' in the diagnosis of myasthenia gravis. A number of small studies have reported improvement of myasthenic ptosis following application of an ice cube, wrapped in a towel or surgical glove, applied to the levator muscle of the eyelid for at least two minutes. ${ }^{4-6}$ The test is claimed to be both sensitive and specific for the diagnosis of myasthenia, ${ }^{56}$ having no effect on ptosis of other cause, such as oculomotor nerve palsy or mitochondrial myopathy. Hence, in view of the difficulty sometimes encountered in establishing a definite diagnosis of ocular myasthenia, the ice pack test is suggested to be of clinical utility. ${ }^{4-6}$ The place of the ice pack test in the differential diagnosis of diplopia remains to be determined. We present a patient with diplopia in whom an ice pack test proved 'positive' but the underlying diagnosis remained unclear.

\section{Case report}

A previously fit 59-year-old man complained of painless diplopia on left lateral and up-gaze of 6 months duration; images were displaced ver-

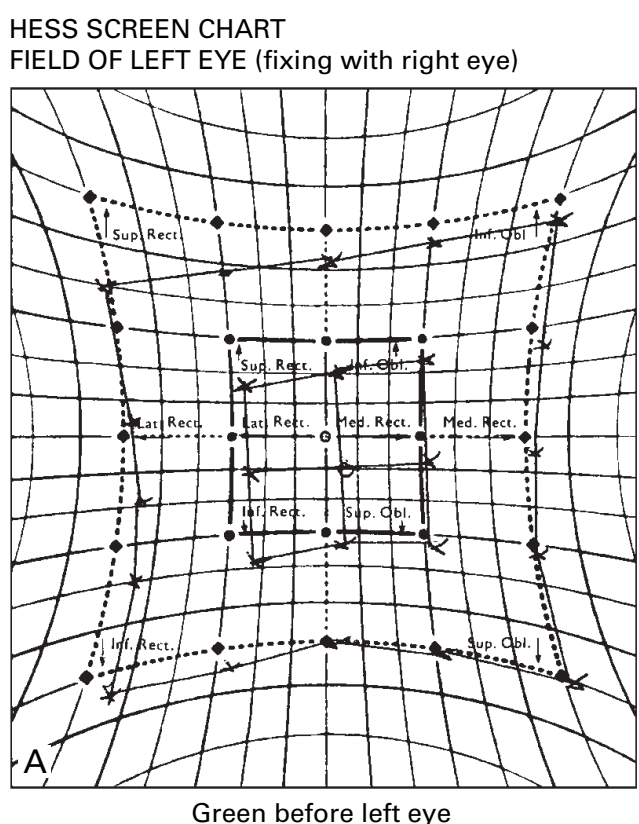

Figure 1 Hess chart, left eye, showing superior rectus underaction

tically. Symptoms tended to be worse in the evening but could be present on waking. There were no bulbar or limb symptoms. On examination there was no ptosis but left hypotropia (superior rectus underaction; figure 1) with no evidence of fatiguability. Neurological examination was otherwise normal. Application of an ice pack to the left eye for 10 minutes led to subjective resolution of the diplopia; Hess chart confirmed an increased action of superior rectus (figure 2). On the basis of this 'positive' ice pack test alone, a diagnosis of ocular myasthenia was made. The patient was commenced on treatment with pyridostigmine and referred to the neurology clinic. AChR autoantibodies were subsequently found to be negative.

Neither pyridostigmine $(60 \mathrm{mg}$ qid for 2 months) nor prednisolone (up to $40 \mathrm{mg}$ on alternate days for 2 months) had any subjective effect on the diplopia, nor did they induce any change in the clinical signs. Electromyography (EMG) of the left orbicularis oculi showed no decrement on repetitive nerve stimulation (3 $\mathrm{Hz}$, train of nine stimuli). Single fibre EMG to study jitter showed a mean consecutive difference (MCD) of time intervals of $31.6 \mu$ s (upper limit, orbicularis oculi MCD at age $60=41.8$
Submitted 5 May 1999 Accepted 13 August 1999 
HESS SCREEN CHART

FIELD OF LEFT EYE (fixing with right eye)

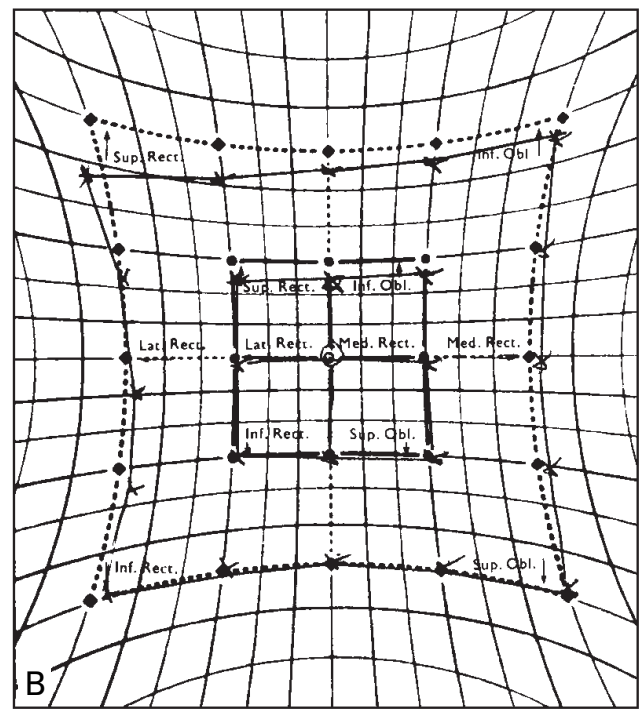

Green before left eye

Figure 2 Hess chart, left eye, after application of ice pack for 10 minutes, showing increased action of superior rectus. There was concurrent subjective resolution of diplopia

$\mu s^{7}$ ) and no blocking was observed. After two weeks off all medication, an intravenous Tensilon (edrophonium chloride) test $(10 \mathrm{mg})$ was performed; there was no subjective improvement and no change was observed in the Hess chart. Subsequent investigations to determine the cause of the diplopia were inconclusive (normal magnetic resonance imaging of the brain, computed tomography of the orbits,

1 Swash M, Schwartz MS. Neuromuscular diseases. A practical approach to diagnosis and management, 3rd edn. Berlin: Springer-Verlag, 1997

2 Simpson JA. Myasthenia gravis: a new hypothesis. Scott Med f 1960;5:419-36.

3 Borenstein S, Desmedt JC. Local cooling in myasthenia Improvement of neuromuscular failure. Arch Neurol 1975; 32:152-7.

4 Saavedra JS, Femminini R, Kochen S, Ortiz de Zarate JC. A cold test for myasthenia gravis. Neurology 1979;29:1075. chest X-ray, thyroid function tests, thyroid autoantibodies).

\section{Discussion}

Previous studies ${ }^{4-6}$ have examined the effect of the ice pack test on ptosis; although involving small numbers of patients, these investigations have suggested that the test is of use in establishing a diagnosis of ocular myasthenia in this situation, both in terms of its sensitivity (compared to the edrophonium test) and specificity. However, the role of the ice pack test in ascertaining the cause of diplopia has not been defined. Saavedra $e t a l^{4}$ state that it had "no definite effect on ocular movement". This result might be expected, since all the rectus muscles have tendinous insertions and thus it is difficult to apply a cold stimulus directly to the muscles themselves, unlike the situation with the levator muscle of the eyelid. In our patient, cooling clearly had a marked subjective and objective (Hess chart) effect on eye movements. This might suggest that the underlying superior rectus underaction was due to myasthenia (certainly very focal and unilateral presentations of myasthenia do occur, albeit rarely $^{8}$ ) but subsequent investigations and treatment provided no evidence to support this diagnosis.

In this case, the ice pack test neither confirmed nor refuted the diagnosis of ocular myasthenia. Contrary to the situation with myasthenic ptosis, systematic studies are still required to determine whether the ice pack test is of use for the diagnosis of myasthenic diplopia. Our experience suggests that, in the assessment of diplopia, caution should at present be exercised in making diagnostic and therapeutic judgements based on its results.

5 Sethi KD, Rivner MH, Swift TR. Ice pack test for myasthenia gravis. Neurology 1987;37:1383-5.

6 Ertas M, Arac N, Kumral K, Tuncbay T. Ice test as a simple diagnostic aid for myasthenia gravis. Acta Neurol Scand 1994;89:227-9.

7 Gilchrist JM, Barkhaus PE, Bril V, et al. Single fiber EMG reference values: a collaborative effort. Muscle Nerve 1992;15:151-61.

8 Janssen JC, Larner AJ, Harris J, Sheean GL, Rossor MN. Myasthenic hand. Neurology 1998;51:913-4. 